\title{
Quantitative Imaging of Sheet Resistance with a Scanning Near-Field Microwave Microscope
}

\author{
D. E. Steinhauer, ${ }^{a)}$ C. P. Vlahacos, S. K. Dutta, B. J. Feenstra, F. C. Wellstood, and Steven M. Anlage \\ Center for Superconductivity Research, Department of Physics, University of \\ Maryland, College Park, MD 20742-4111
}

\begin{abstract}
We describe quantitative imaging of the sheet resistance of metallic thin films by monitoring frequency shift and quality factor in a resonant scanning near-field microwave microscope. This technique allows fast acquisition of images at approximately $10 \mathrm{~ms}$ per pixel over a frequency range from 0.1 to $50 \mathrm{GHz}$. In its current configuration, the system can resolve changes in sheet resistance as small as $0.6 \Omega / \square$ for $100 \Omega / \square$ films. We demonstrate its use at $7.5 \mathrm{GHz}$ by generating a quantitative sheet resistance image of a $\mathrm{YBa}_{2} \mathrm{Cu}_{3} \mathrm{O}_{7-\delta}$ thin film on a $5 \mathrm{~cm}$-diameter sapphire wafer.
\end{abstract}

Non-destructive imaging of microwave sheet resistance has been demonstrated using a variety of probes combined with resonant systems. Far-field techniques, such as confocal垉 and dielectric四 resonators, while allowing quantitative sheet resistance imaging, have the disadvantage of relatively low spatial resolution ( $\gtrsim 1 \mathrm{~mm})$ limited by the wavelength. Near-field microscopy using coaxial, $\mathrm{d}$ microstrip, $\mathrm{U}$ or waveguidd $\mathrm{\theta}$ resonators, offers higher spatial resolution. However, current techniques either require contact with the sample inhibiting quantitative interpretation of the data, have low sheet resistance sensitivity in conducting samples, wavelength resolution. 8 For non-destructive sheet resistance imaging of thin films, it is desirable to have quantitative methods that combine high resolution, high speed, simple construction from commercially-available components, and straightforward image interpretation. We describe here the application of an open-ended coaxial probe resonator to obtain quantitative images of microwave sheet resistance with $\lambda / 80$ spatial resolution.

Our scanning microwave microscope consists of resonant coaxial transmission line connected to an open-ended coaxial probe and a microwave source (Fig. 1. 1012 The microwave source, which is weakly coupled to the resonant transmission line through a decoupling capacitor $C_{D}$, is frequency modulated by an external oscillator at a rate $f_{F M} \sim 3 \mathrm{kHz}$. The electric field at the probe tip is perturbed by the region of the sample beneath the probe's center conductor. We monitor these perturbations using a diode-detector which produces a voltage signal proportional to the power reflected from the resonator. A feedback circuit 12 (Fig. 11) keeps the microwave source locked to a resonant frequency of the transmission line, and has a voltage output which is proportional to shifts in the resonant frequency due to the sample.

To determine the quality factor $\mathrm{Q}$ of the resonant circuit, a lock-in amplifier, referenced at $2 f_{F M}$, gives an output voltage $V_{2 f_{F M}}$ which is related to the curvature of the reflected power-vs.-frequency curve on resonance, and hence to $\mathrm{Q}$. To relate $V_{2 f_{F M}}$ and $\mathrm{Q}$, we perform a separate experiment, in which we vary $\mathrm{Q}$ using a microwave absorber at various heights below the probe tip, and measure the absolute reflection coefficient $|\rho|^{2}$ of the resonator. If $\left|\rho_{0}\right|^{2}$ is the reflection coefficient at a resonant frequency $f_{0}$, then the coupling coefficient between the source and the resonator is $\beta=\left(1-\left|\rho_{0}\right|\right) /\left(1+\left|\rho_{0}\right|\right)$. The loaded quality factor of the resonator 13 is $\mathrm{Q}_{L}=f_{0} / \Delta f$, where $\Delta f$ is the difference in frequency between the two points where $|\rho|^{2}=\left(1+\beta^{2}\right) /(1+\beta)^{2}$. The unloaded quality factor, which is the $\mathrm{Q}$ of the resonator without coupling to the microwave source and detector, is then $\mathrm{Q}_{0}=\mathrm{Q}_{L}(1+\beta)$. We also measure $V_{2 f_{F M}}$, and find that there is a unique functional relationship between $\mathrm{Q}_{0}$ and $V_{2 f_{F M}}$; thus, we need to calibrate this relationship only once for a given microscope resonance. In a typical scan, we record $V_{2 f_{F M}}$, and afterward convert $V_{2 f_{F M}}$ to $\mathrm{Q}_{0}$.

To determine the relationshim between $\mathrm{Q}_{0}$ and sample sheet resistance $\left(R_{X}\right)$, we used a variable-thickness aluminum thin film on a glass substrate.12 The cross-section of the thin film is wedge-shaped, implying a spatially varying sheet resistance. Using a probe with a $500 \mu \mathrm{m}$ diameter center conductor, and selecting a resonance of the microscope with a frequency of $7.5 \mathrm{GHz}$, we acquired frequency-shift and $\mathrm{Q}_{0}$ data. We then cut the sample into narrow strips to take two-point resistance measurements and determine the local sheet resistance. The unloaded $\mathrm{Q}_{0}$ of the resonator as a function of $R_{X}$ is shown in Fig. 2 for various probe-sample separations. We note that $\mathrm{Q}_{0}$ reaches a maximum as $R_{X} \rightarrow 0$; as $R_{X}$ increases, $Q_{0}$ drops due to loss from currents induced in the sample, reaching a minimum around $R_{X}=660 \Omega / \square$ for a height of $50 \mu \mathrm{m}$. Similarly, as $R_{X} \rightarrow \infty, \mathrm{Q}_{0}$ increases due to diminishing currents in the sample.

a) Electronic mail: steinhau@squid.umd.edu. Color versions of the figures in this paper can be found at http://www.csr.umd.edu/research/hifreq/micr_microscopy.html. 
We also note that when the probe is located $50 \mu \mathrm{m}$ above the bare glass substrate, $\mathrm{Q}_{0}=549$, which is only slightly less than $\mathrm{Q}^{\prime}=555$ when the probe is far away $(>1 \mathrm{~mm})$ from the sample. Using $1 / \mathrm{Q}_{0}=1 / \mathrm{Q}_{s}+1 / \mathrm{Q}^{\prime}$, we find $\mathrm{Q}_{s}=51000 \gg \mathrm{Q}^{\prime}=555$, where $\mathrm{Q}_{s}$ is associated with losses in the glass substrate, and $\mathrm{Q}^{\prime}$ is associated with losses in the transmission line. As a result, we conclude that the glass substrate has little effect on $Q_{0}$. In contrast, because the dielectricsubstrate effectively lengthens the microscope resonant circuit, frequency shift is highly sensitive to the substrate.12.16 This suggests that we use the Q data, rather than the frequency shift data, to generate substrateindependent images of thin film sheet resistance.

As shown in Fig. 2, $R_{X}$ is a double-valued function of $Q_{0}$. This presents a problem for converting the measured $Q_{0}$ to $R_{X}$. However, $R_{X}$ is a single-valued function of the frequency shift 12 allowing one to use the frequency shift data to determine which branch of the $R_{X}(\mathrm{Q})$ curve should be used.

To better understand the system's behavior, we modeled the interaction between the probe and sample as a capacitance $G_{\text {b }}$ between the probe's center conductor and the sample in series with the sample sheet resistance $R_{X}$ (see Fig. 1) 12.16 We assumed a parallel plate approximation for the capacitor $C_{X}$, and took $R_{X}=\tilde{\rho} / t, 12$ where $\tilde{\rho}$ is the sample dc resistivity, and $t$ is the film thickness. To find $\mathrm{Q}_{0}$, we calculated the width of the resonant minimum in the reflection coefficient $|\rho|^{2}$ vs. frequency curve, as described above. The model results for a height of $50 \mu \mathrm{m}$ are indicated by the solid line in Fig. 2. To fit the data we used the measured $\mathrm{Q}_{0}=555$ and $\mathrm{Q}_{L}=353$ with the probe far away from the sample to fix two fitting parameters: the coupling capacitance $C_{D}=0.17 \mathrm{pF}$, and the ransmission line attenuation constant $\alpha=-1.26 \mathrm{~dB} / \mathrm{m}$ (in close agreement with the manufacturer's specified value 15 of $\alpha=-1.23$ $\mathrm{dB} / \mathrm{m})$. Aside from a coupling factor involving $C_{X}$, the model agrees qualitatively and predicts the $R_{X}$ which yields the minimum $\mathrm{Q}_{0}$ to within $10 \%$. For large $R_{X}$, the model predicts a faster return to the asymptotic $\mathrm{Q}_{0}$ value of 555 . The overall behavior of this simple model confirms our understanding of the system.

To explore the capabilities of our system, we scanned a thin film of $\mathrm{YBa}_{2} \mathrm{Cu}_{3} \mathrm{O}_{7-\delta}$ (YBCO) on a 5 cm-diameter sapphire substrate at room temperature. The film was deposited using pulsed laser deposition with the sample temperature controlled by radiant heating. The sample was rotated about its center during deposition, with the $\sim 3$ $\mathrm{cm}$ diameter plume held at a position halfway between the center and the edge. The thickness of the YBCO thin film varied from about $100 \mathrm{~nm}$ at the edge to $200 \mathrm{~nm}$ near the center.

Figure 3 shows three microwave images of the YBCO sample. The frequency shift [Fig. 3(a)] and $Q_{0}$ [Fig. 3(b)] were acquired simultaneously, using a probe with a $500 \mu \mathrm{m}$-diameter center conductor at a height of $50 \mu \mathrm{m}$ above the sample. The scan took approximately 10 minutes to complete, with raster lines $0.5 \mathrm{~mm}$ apart. The frequency shifts in Fig. 3(a) are relative to the resonant frequency of $7.5 \mathrm{GHz}$ when the probe was far away ( $>1 \mathrm{~mm})$ from the sample; the resonant frequency shifted downward by more than $2.2 \mathrm{MHz}$ when the probe was above the center of the sample. Noting that the resonant frequency drops monotonically between the edge apd the center of the film, and that the resonant frequency is a monotonically increasing function of sheet resistance 12 we conclude that the sheet resistance decreases monotonically between the edge and the center.

The frequency shift and $Q_{0}$ images [Fig. 3(a) and (b)] differ slightly in the shape of the contour lines. This is most likely due to the $300 \mu \mathrm{m}$-thick substrate being warped, causing a variation of a few microns in the probe-sample separation during the scan. However, for a sample such as that shown in Fig. 3, with an $R_{X}$ variation across the sample of $\sim 100 \Omega / \square$, the $Q_{0}$ data are primarily sensitive to changes in $R_{X}$, while the frequency shift data are primarily sensitive to changes in probe-sample separation. As a result, we attribute the difference between the frequency shift and $\mathrm{Q}_{0}$ images to small changes in probe-sample separation, which will mainly affect the frequency shift data. Since the values of $R_{X}$ are retrieved from the $\mathrm{Q}$ data, the warping does not affect the final $R_{X}$ appreciably. In principle, one can use the combined frequency shift and Q images to extract sample topography information, 16 allowing one to separate the effects of sample sheet resistance and topography.

From Fig. 3(b), we see that the lowest Q occurs near the edge of the film, and that the Q rises toward the center of the sample. As mentioned above, $R_{X}$ is not a single-valued function of $\mathrm{Q}$ and we must use the frequency shift image [Fig. 3(a)] to determine which branch of the $Q_{0}$ vs. $R_{X}$ curve in Fig. 2 to use. From the frequency shift image we learned that $R_{X}$ decreases monotonically from the edge to the center of the sample; therefore we use the branch of the $\mathrm{Q}_{0}$ vs. $R_{X}$ curve with $R_{X}<660 \Omega / \square$, since this is the branch that yields a decreasing $R_{X}$ for increasing $\mathrm{Q}_{0}$.

With the appropriate branch identified, we then transformed the $\mathrm{Q}$ image in Fig. B(b) to the sheet resistance image in Fig. B (c) using a polynomial least-squares fit to the data presented in Fig. 2 for $\mathrm{R}_{X}<540 \Omega / \square$ and a height of $50 \mu \mathrm{m}$. Figure 3(c) confirms that the film does indeed have a lower resistance near the center, as was intended when the film was deposited. We note that the sheet resistance does not have a simple radial dependence, due to either non-stoichiometry or defects in the film.

After scanning the YBCO film, we patterned it and made four-point dc resistance measurements all over the wafer. The dc sheet resistance had a spatial dependence identical to the microwave data in Fig. 3(c). However, the absolute values were approximately twice as large as the microwave results, most likely due to degradation of the film during patterning.

To estimate the sheet resistance sensitivity, we monitored the noise in $V_{2 f_{F M}}$. We find the $\mathrm{Q}$ sensitivity of the 
system to be $\Delta \mathrm{Q}_{0} \approx 0.08$ for $\mathrm{Q}_{0}=555$ and an averaging time of $10 \mathrm{~ms}$. Combining this with the data in Fig. 2, we find $\Delta R_{X} / R_{X}=6.4 \times 10^{-3}$, for $R_{X}=100 \Omega / \square$ using a probe with a $500 \mu \mathrm{m}$ diameter center conductor at a height of $50 \mu \mathrm{m}$ and a frequency of $7.5 \mathrm{GHz}$. The sensitivity scales with the capacitance between the probe center conductor and the sample $\left(C_{X}\right)$; increasing the diameter of the probe center conductor and/or decreasing the probe-sample separation would improve the sensitivity.

In conclusion, we have demonstrated a technique which uses a near-field microwave microscope to generate quantitative sheet resistance images of thin film samples. The strengths of our system include the ability to arrive at quantitative results and to confirm our understanding of the system with a simple model. Other advantages include its speed, measurement frequency bandwidth, construction from standard commercially-available components, and the possibility of enhancing its spatial resolution by using a probe with a smaller-diameter center conductor. 10

The authors would like to thank Alberto Pique of Neocera, Inc., for the YBCO wafers. This work has been supported by NSF-MRSEC grant No. DMR-9632521, NSF grants No. ECS-9632811 and DMR-9624021, and by the Center for Superconductivity Research.

${ }^{1}$ Steve Hogan, Sigurd Wagner, and Frank S. Barnes, Appl. Phys. Lett. 35, 77 (1979).

${ }^{2}$ J. S. Martens, V. M. Hietala, D. S. Ginley, T. E. Zipperian, and G. K. G. Hohenwarter, Appl. Phys. Lett. 58, 2543 (1991).

3 John Gallop, Ling Hao, and Farhat Abbas, Physica C 282-287, 1579 (1997).

${ }^{4}$ C. Wilker, Z-Y. Shen, V. X. Nguyen, and M. S. Brenner, IEEE Trans. Appl. Supercond. 3, 1457 (1993).

${ }^{5}$ C. A. Bryant and J. B. Gunn, Rev. Sci. Instr. 36, 1614 (1965).

${ }^{6}$ I. Takeuchi, T. Wei, Fred Duewer, Y. K. Yoo, X.-D. Xiang, V. Talyansky, S. P. Pai, G. J. Chen, and T. Venkatesan, Appl. Phys. Lett. 71, 2026 (1997).

${ }^{7}$ Massood Tabib-Azar, Neil S. Shoemaker, and Stephen Harris, Meas. Sci. Technol. 4, 583 (1993).

${ }^{8}$ M. Golosovsky, A. Galkin, and D. Davidov, IEEE Trans. Microwave Theor. Tech., 44, 1390 (1996).

${ }^{9}$ Yansheng Xu and R. G. Bosisio, IEE Proc.-H 139, 500 (1992).

${ }^{10}$ C. P. Vlahacos, R. C. Black, S. M. Anlage, A. Amar, and F. C. Wellstood, Appl. Phys. Lett. 69, 3274 (1996).

11 S. M. Anlage, C. P. Vlahacos, Sudeep Dutta, and F. C. Wellstood, IEEE Trans. Appl. Supercond. 7, 3686 (1997).

12 D. E. Steinhauer, C. P. Vlahacos, S. K. Dutta, F. C. Wellstood, and Steven M. Anlage, Appl. Phys. Lett. 71, 1736 (1997), cond-mat/9712142.

13 J. E. Aitken, Proc. IEE 123, 855 (1976).

${ }^{14}$ K. Zaki and G. J. Chen, private communication.

${ }^{15}$ Private communication with W. L. Gore and Assoc., Inc. (see also reference 12 of reference 12).

${ }^{16}$ C. P. Vlahacos, D. E. Steinhauer, S. K. Dutta, B. J. Feenstra, Steven M. Anlage, and F. C. Wellstood, submitted to Appl. Phys. Lett. 


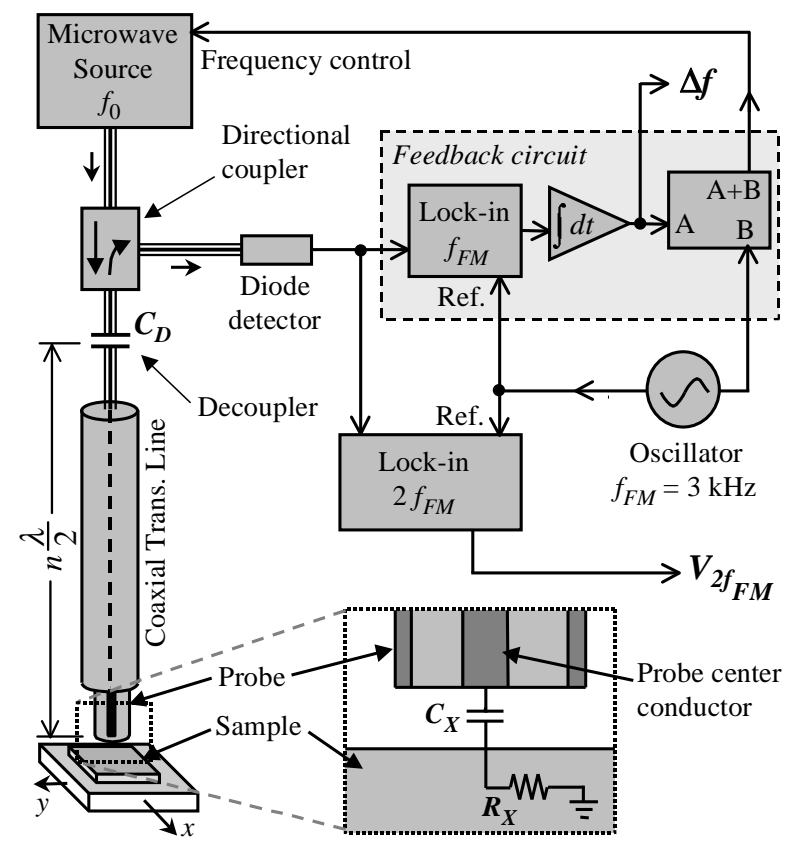

FIG. 1. Schematic of the scanning near-field microwave microscope. The inset shows the interaction between the probe and the sample, represented by a capacitance $C_{X}$ and a resistance $R_{X}$. 


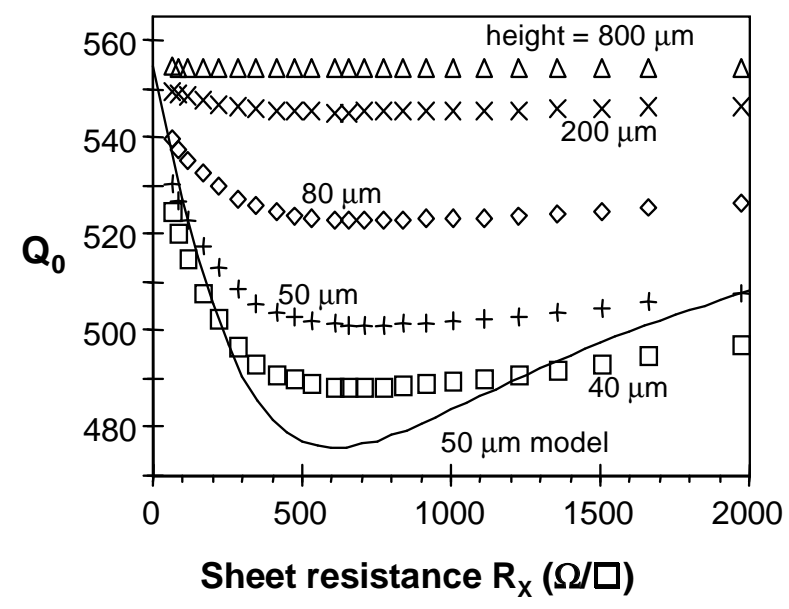

FIG. 2. Unloaded quality factor $Q_{0}$ of the resonant circuit as a function of the sheet resistance $R_{X}$ of a variable-thickness aluminum thin-film sample. The labels indicate different probe-sample separations. A probe with a $500 \mu \mathrm{m}$ center conductor was used at a frequency of $7.5 \mathrm{GHz}$. The solid line indicates a model calculation for a probe-sample separation of $50 \mu \mathrm{m}$. 
(a)

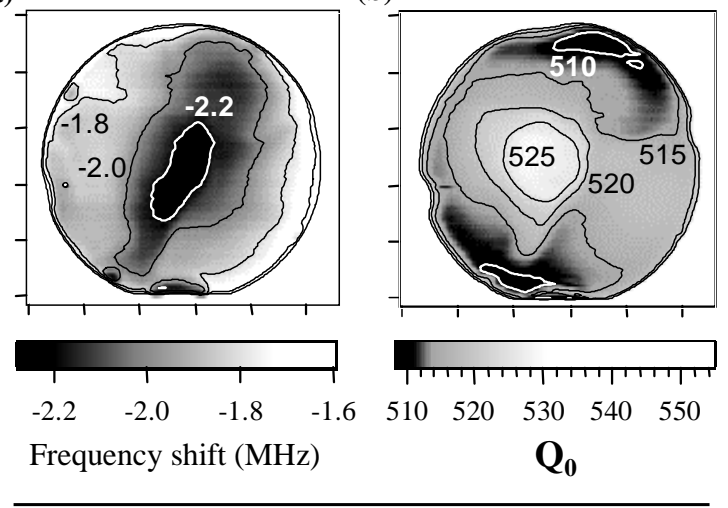

(c)

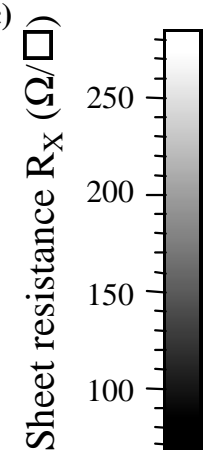

(b)

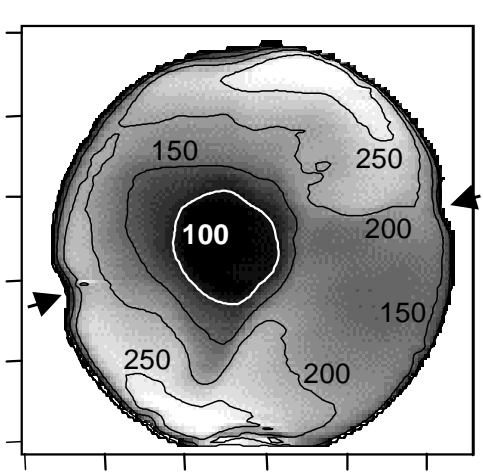

FIG. 3. Images of a variable-thickness YBCO thin-film on a $5 \mathrm{~cm}$-diameter sapphire wafer, where the film is the thickest at the center. The tick marks are $1 \mathrm{~cm}$ apart for the images of (a) frequency shift relative to the resonant frequency when the probe is far away $\left(>1 \mathrm{~mm}\right.$ ) from the sample, (b) unloaded Q, and (c) sheet resistance $\left(R_{X}\right)$. The arrows in (c) point to small semi-circular regions where clips held the wafer during deposition, and thus no film is present. The labels indicate values at each contour line. A probe with a $500 \mu \mathrm{m}$ diameter center conductor was used at a height of $50 \mu \mathrm{m}$, at a frequency of $7.5 \mathrm{GHz}$. 
This figure "figure1.gif" is available in "gif" format from: http://arxiv.org/ps/cond-mat/9712171v1 
This figure "figure2.gif" is available in "gif" format from: http://arxiv.org/ps/cond-mat/9712171v1 
This figure "figure3.gif" is available in "gif" format from: http://arxiv.org/ps/cond-mat/9712171v1 